\title{
HACIA UNA CARACTERIZACIÓN DEL DOCENTE UNIVERSITARIO "EXCELENTE": UNA REVISIÓN A LOS APORTES DE LA INVESTIGACIÓN SOBRE EL DESEMPEÑO DEL DOCENTE UNIVERSITARIO
}

\author{
Susan Francis Salazar ${ }^{1}$ \\ Directora del Departamento de Docencia Universitaria de la \\ Escuela de Formación Docente de la Universidad de Costa.
}

\begin{abstract}
Resumen: El presente artículo muestra un análisis de las cualidades del profesor universitario efectivo que emergen del estudio e investigación sobre la evaluación del desempeño docente en la educación superior. En el estudio de las características del docente universitario emergen dos grandes tendencias: la declaración de perfiles en artículos de reflexión y ensayo y los procesos de evaluaciones del desempeño docente. El propósito de concentrarse en esta última tendencia surge en la posibilidad de encontrar significativos aportes para la comprensión del quehacer del profesor universitario por su relación con la realidad empírica en la que el docente está inmerso. El análisis de estos estudios muestra al menos dos categorías de estudio: la multidimensionalidad del docente $y$ el valor histórico y contextual de las cualidades que lo hacen un profesor o profesora de calidad. El foco de este artículo es la primer categoría que revela la interrelación de tres ámbitos: el personal, el disciplinar y el pedagógico, para que sirva de insumo en la discusión e investigación de los procesos formativos del profesor y profesora universitaria.
\end{abstract}

Palabras clave: Evaluación docente, efectividad en la enseñanza, formación del docente universitario, el docente universitario efectivo.

\section{Introducción}

La actividad del docente universitario es compleja: incorpora elementos personales, disciplinares y pedagógicos. Su tarea primordial es el acto educativo dentro de su disciplina, por ello, tiene exigencias en el bagaje de conocimientos disciplinares y en lo pedagógico, o sea, en cómo encontrar formas interventivas que permitan enseñar aquellos conocimientos, habilidades, destrezas y valores que deben aprender los próximos profesionales. También deben buscar cómo van a ser aprehendidos estos contenidos, en los contextos en que profesores y estudiantes interactúan y construyen una dinámica particular.

La comprensión del acto docente universitario exige un reconocimiento de su complejidad por la vinculación con lo político, institucional, disciplinar, pedagógico y lo macrosocial. (Venegas, 1995). La docencia universitaria se desarrolla en un contexto de academia y en un entramado de relaciones de poder, generación de investigación y extensión universitaria, demandas de retorno social, presiones económicas, 


\begin{abstract}
This article presents an analysis of the qualities of an effective college professor. The analysis emerges from research on the professor's performance assessment. In conducting this type of research, it is possible to identify two tendencies: (1) a reflection and essay articles about the profiles of effectiveness and (2) research and performance assessment process. The focus is on the latter tendency in order to find out the basis to for understand the college professors' action in their relationship with the context The analysis of these studies at least shows two categories: the professor's multi-dimension and historical and contextual value of qualities that make this professor effective. The first tendency seeks to reveal the interrelation between the personal, disciplinary and pedagogical scopes. The third scope is very important in terms of the research on orientations in the process of training college professors.
\end{abstract}

Key words: Teacher evaluation, effective teaching, training college professors, effective college professors. en fin, la misma universidad es el reflejo de la sociedad, de sus actividades y de sus transformaciones. Por tanto, una aproximación para determinar los elementos que orienten la formación y la evaluación del quehacer del docente universitario requiere del análisis del contexto en el cual surgen y se desarrollan.

El docente universitario ha sido concebido como un especialista de alto nivel, dedicado a la enseñanza y miembro de una comunidad académica. Esto implica que comparte la tarea del logro de los aprendizajes en otros, puesto que en conjunto con otros especialistas asumen como responsabilidad la formación de nuevos profesionales dentro de su disciplina. También está ávido de capacidad y posee hábitos investigativos que le permiten el acercamiento científico a su objeto de estudio disciplinar, específicamente en su estructura sustantiva que incluye la variedad de formas en las cuales los conceptos básicos y principios teóricos son organizados para explicar la realidad. No obstante, estos procesos investigativos no necesariamente consideran la dimensión pedagógica que le es propia a los distintos objetos de estudio disciplinar. (Francis, 2005). Por último, el docente universitario pertenece a una comunidad académica en la cual comparte, reconstruye y sigue pautas, valores y actitudes que configuran su visión y accionar en el mundo.

Las exigencias que se evidencian en los distintos discursos sobre el papel del docente incluyen todos los niveles educativos formales, inclusive el universitario. Por ejemplo, de acuerdo con GarcíaValcárcel (2001:10) las demandas a nivel del desarrollo de las naciones redimensionan el papel docente universitario y le exigen convertirse en especialista en diagnóstico y prescripción del aprendizaje, especialista en recursos de aprendizaje, facilitador del aprendizaje en comunidad, especialista en la convergencia interdisciplinar de saberes, clasificador de valores, 
promotor de relaciones humanas y en consejero profesional y del ocio.

La UNESCO (1998) señala que el profesor universitario debe reunir como competencias: el conocimiento y entendimiento de los distintos modos de aprendizaje de los estudiantes ${ }^{2}$, conocimientos, competencias y aptitudes en materia de evaluación de los aprendizajes, a fin de ayudarles a aprender; el compromiso con el saber en la disciplina en el marco del respeto de las normas profesionales y del conocimiento de las nuevas circunstancias; el conocimiento de las aplicaciones de la ciencia y la tecnología a la disciplina, en relación con el acceso al material y los recursos en el ámbito mundial y con la enseñanza de la tecnología; receptividad a los indicios del "mercado exterior" para conocer las necesidades de aquellos que podrían contratar a los graduados de una disciplina; dominio de las innovaciones en el campo de la enseñanza, incluida la conciencia de la necesidad de una instrucción de "modo doble" en la que la educación presencial y a distancia emplean un material similar; conocimientos de los usuarios, en particular las opiniones y aspiraciones de los interesados, entre ellos los estudiantes; conciencia de la influencia que ejercerían los factores internacionales y multiculturales sobre los planes de estudios; capacidad de instruir a una amplia gama de estudiantes, de distintos grupos de edad, origen socioeconómico, raza, etc., a lo largo del día; aptitudes para ocuparse de un número mayor de estudiantes en las clases, los seminarios o talleres formales, conservando la calidad, elaboración de "estrategias de gestión" personales y profesionales.

$\mathrm{Al}$ respecto, es importante hacer la advertencia que plantea Torres (1998) al indicar que la "lógica de los listados" destaca el simplismo respecto del cambio educativo, su pretendida validez universal, así como el hecho de que se limitan a describir un conjunto de características deseables; pero no proveen elementos que ayuden a definir los caminos para construir esas características en situaciones concretas. Por ejemplo, la UNESCO hace este llamado de atención en términos genéricos sin atender las diferencias entre los objetos de estudio disciplinares. La constitución de extensas listas de características sobre el docente universitario "efectivo" ha desatado reacciones que dan cuenta de un desconocimiento del contexto de las prácticas del docente y de una verdadera lejanía de la realidad, de ahí la imposibilidad de ver concretadas estas competencias en nuestras universidades.

La mayoría de esfuerzos por definir propuestas de perfiles para el docente universitario se encuentran en artículos de reflexión y ensayos. Muy pocos aportes surgen de la investigación (Campo, 2001). Los procesos investigativos acerca del profesor universitario son recientes y se han potenciado a raíz de los procesos de acreditación y evaluación universitaria (Alvarez, García y Gil, 1999). Sus resultados confirman las críticas a los enfoques pedagógicos desarrollados en el aula universitaria y el valor de las creencias en la configuración del enfoque pedagógico promovido por el docente, o sea, las formas en las cuales piensa, organiza, desarrolla y valora el acto educativo son determinantes, pues se ha demostrado que explican la separación y falta de congruencia entre los planos discursivos, prescriptitos y configurados en la práctica y, sin embargo, no han sido consideradas para plantear la transformación en el aula. ${ }^{3}$

La observación y análisis de los estudios sobre las características de los docentes en espacios reales, provee un espacio importante para desarrollar investigación que fundamente acciones formativas sistematizadas para el docente universitario, tanto de lo disciplinar como de lo pedagógico, asimismo dentro de su comunidad disciplinar y de una comunidad académica universitaria.

Considerando los retos que enfrenta el docente universitario de cara a la evolución 
de los tiempos, la necesidad de encontrar formas de apoyo sistemático que permitan generar bases para formación académica para el docente universitario que observen los contextos e historicidad de los procesos docentes académicos, se propone el presente artículo con el propósito de presentar la revisión y análisis de resultados de investigaciones sobre la constitución de factores que caractericen al docente universitario con éxito en el quehacer del aula.

Se busca ante todo revisar y analizar el carácter multidimensional para comprender la complejidad del acto docente universitario y responderse: ¿Cuáles dimensiones explican la efectividad del docente universitario, según los procesos investigativos y evaluativos? ¿Qué relaciones se pueden identificar entre estas dimensiones? ¿Qué categorías conforman cada una de las dimensiones? ¿Cuál es el valor de los aportes de estos estudios para la constitución de bases para programas de apoyo formativo académico para docentes universitarios?

Para cumplir con este propósito se han seleccionado reportes de investigación que incluyen estudios de evaluación del desempeño docente universitario, dentro de los cuales, encontramos dos categorías: los basados en el enfoque empírico-analítico ${ }^{4}$; desarrollados desde la década de los ochenta hasta la fecha; y los de investigaciones con un enfoque histórico-hermenéutico ${ }^{5}$, las cuales son más recientes (los reportes datan de 1995 a la fecha) y más que evaluar el desempeño docente, profundizan en las características que presentan los profesores y las profesoras con mayor calidad en su acción pedagógica en el ámbito universitario.

Una reflexión importante sobre lo anterior, es que gran parte de las decisiones pedagógicas que le permiten al docente universitario seleccionar las formas interventivas, con las cuales espera que los estudiantes aprehendan los contenidos disciplinares, están fundamentalmente basadas en experiencias anteriores no sistematizadas, como estudiante y docente, puesto que los docentes universitarios no requieren procesos sistemáticos de formación profesional para el ejercicio docente. Esto significa que no necesariamente acuden a la investigación educativa para identificar formas de actuación docente ni formas de construcción de determinados saberes profesionales. Empero el docente universitario tiene un alto nivel de responsabilidad en su labor educativa.

\section{Conceptuando la "excelencia" en la docencia universitaria}

La caracterización del docente excelente o del mejor docente no puede concebirse sin entender que cada docente se construye en un contexto que le hace particular. Por un lado, por los significados que se sitúan en los espacios asociados al ámbito universitario y, por otro, por la cultura organizacional construida y reflejada en cada una de las facultades de formación profesional.

Para los efectos de este artículo se define como docente universitario "excelente" aquel docente que desarrolle un modelo docente configurado por formas de actuación que satisfacen las necesidades y expectativas de formación profesional de los estudiantes universitarios. (Álvarez; García; Gil, 1999). Entendiendo que el espacio universitario es complejo y transpira una diversidad cultural que se refleja en los distintos sujetos que la conforman, esta diversidad también se hace evidente en sus procesos y prácticas cotidianas universitarias.

Los perfiles de calidad docente son una forma de categorizar cualidades ideales de un profesor universitario, estos se identifican, sobre todo, con la aparición de mecanismos de evaluación y acreditación institucional. (Quirós y Jiménez 1991; Álvarez, García y Gil 1999). Parte de los perfiles del docente universitario califican al docente que cumple con todos los criterios establecidos como "excelente", "el buen 
profesor", "el maestro eficiente" o "el profesor mejor valorado".

Existe una producción sobre perfiles del docente universitario que, como ya se citó, surge de propuestas retóricas y se asocian más con el deber ser. Esta producción se orienta hacia la prescripción de las acciones del docente universitario en su labor, no sólo en el aula, sino además como investigador y promotor de la transformación social.

Otros perfiles han surgido como producto de los estudios que han desarrollado propuestas de evaluación del desempeño del docente desde el estado teórico de la docencia en general ${ }^{6}$. La revisión de perfiles y evaluaciones del desempeño del docente universitario sugiere que su construcción implica considerar la tarea y el ejercicio académico como aquel que trasciende la enseñanza y abarca cualidades y atributos que le son particulares a la educación superior, entre ellos el trabajo formal de investigación y de extensión docente que por tanto, la hacen ser más extensa con respecto a la docencia en general.

La función docente incorpora en una triple dimensión de saberes profesionales -académicos, de investigación y de formación-, los que se traducen como una trilogía de ser, saber conocer y saber hacer. El primero incorpora los aspectos axiológicos y éticos y enmarca los determinantes actitudinales y valorativos; los segundos, más reconocidos en el perfil del docente universitario, están condicionados por la disciplina que se enseña y en la que se reconoce una heterogeneidad y diferenciación en el contenido, su estructura como conocimiento; y por último, los terceros abarcan las habilidades y procesos intelectuales que le son propios a su disciplina y que en consecuencia involucran su dimensión pedagógica. (Quirós y Jiménez, 1991).

Sin embargo, el cumplimiento de estos perfiles se enfrenta a la distancia que prevalece en las prescripciones puesto que, en ocasiones, tienden a generalizaciones que no son sensibles a los distintos espacios académicos (sub-mundos para Berger \& Luckman; 2003, sub-culturas para Robbins; 1994) de acuerdo con cada facultad.

De ahí el valor de reconocer las cualidades de excelencia del docente al recurrir a la evaluación que considera la recolección de información de las realidades contextuales a cada ejercicio docente. El desarrollo de la evaluación de la calidad docente en la educación superior, sobre todo en los Estados Unidos, Australia y Europa; particularmente en Gran Bretaña, evidencian un desarrollo teórico y metodológico importante, que ofrece elementos que pueden orientar la investigación del docente universitario y en particular del docente excelente ${ }^{7}$. Estos elementos son discutidos más adelante con el fin de identificar los aportes y pertinencia con la realidad costarricense.

\section{Las dimensiones que explican "la excelencia" del docente universitario y su quehacer}

De la revisión y análisis de los reportes investigativos surgen dos categorías de análisis:

- El carácter histórico de las construcciones acerca del concepto de docente universitario y de las configuraciones de la práctica del profesor universitario $\mathrm{y}$,

- La multidimensionalidad de este concepto, su construcción así como del mismo ejercicio docente.

El reconocimiento del carácter histórico en la constitución de la práctica docente surge como comprensión de que la actividad humana es el reflejo de entramados sociales e individuales, que interactúan dialógicamente, en un contexto y un momento histórico en la cual está inserta y la determina, así lo pedagógico refleja las interpretaciones que el docente hace de los entramados según los momentos y el espacio en el que se encuentre. ${ }^{8}$ 
Otro elemento sustantivo es el carácter multidimensional de la tarea docente. El cuadro 1 revela los rasgos que han explicado la excelencia como resultado de procesos investigativos (Alvarez, García y Gil, 1999; Young \& Shawn, 1999; Tsui, 2002; Campo, 2001; Stronge, 2002; Corbett \& Wilson, 2002). Los resultados permiten identificar tres dimensiones en la tarea docente efectiva: la personal, la pedagógica y la disciplinar, estas tienen una interacción constante que va modelando la función docente. Ninguna de las investigaciones analizadas señala una jerarquía que otorgue primacía a alguna dimensión en particular.

\section{La dimensión personal}

La dimensión personal incorpora las acciones asociadas al ámbito de empatía en las relaciones que establece el profesor con sus estudiantes. La significatividad de esta dimensión se reconoce en los altos coeficientes de correlación mostrados con respecto al logro educacional pretendido. Cashin (1988) reportó, después de analizar diversos estudios correlacionales, que el aprendizaje de los estudiantes y las relaciones interpersonales que establece el docente tienen una correlación de 0,31, la cual considera "prácticamente útil" por ser un objeto de estudio complejo y perteneciente a las ciencias sociales. Marsh (1990) por su parte, señala que el entusiasmo mostrado por el docente, en el acto educativo, presenta correlaciones que van desde 0,43 a 0,319 con cada uno de los siguientes elementos: el valor del aprendizaje, la claridad y organización de la clase, las interacciones grupales, con la interacción personal, amplitud de los conocimientos, las evaluaciones y calificación, las lecturas y trabajos asignados. Y sólo presenta una correlación baja de 0,07 con la carga de trabajo y su dificultad. Los resultados reportados por Young y Shaw (1999) indican que entre las características predictoras en un perfil de un docente efectivo se encuentra "la motivación hacia los estudiantes para hacer su mejor trabajo" con un coeficiente $\mathrm{R}^{2}$ de 0,796 y "la comunicación efectiva" con un coeficiente $R^{2}$ de 0,863, por otro lado al aplicar una función discriminante ${ }^{9}$ aparece "el respeto hacia los estudiantes" con una media de 7,94 en una escala de 1 a 10 .

El docente como persona ve reflejada su condición humana en sus relaciones, mismas que explicitan los valores con que comulga y que se asocian con las creencias y teorías implícitas que marcan las rutas de conversación, trato y aceptación hacia otros. La coherencia entre el discurso del deber ser como persona y su praxis como docente es un requisito tanto deseable como indispensable (Campo, 2001) porque el docente señala lo que espera que aprendan los estudiantes y modela valores y calidades comportamentales que también son aprehendidas por los estudiantes. Los rasgos dentro de esta dimensión se pueden categorizar de acuerdo con el ámbito de relación personal. Según lo anterior se pueden citar cuatro categorías: la interacción docente, los valores asociados a la interacción docente, la promoción del aprendizaje y la actitud hacia la docencia.

\section{a. La interacción docente-estudiante}

Dentro de los reportes de investigación analizados se señalan dos características fundamentales: el rol de cuidado y las interacciones con los estudiantes (Stronge, 2002). El rol de cuidado se define como la presencia de acciones de escucha, comprensión y conocimiento del estudiante. Para este caso la escucha se refiere a la acción docente de poner atención para entender lo que el estudiante dice. Esta atención se focaliza en el estudiante que habla, se constituye en una comunicación en el sentido estricto de la palabra, puesto que existe una relación 


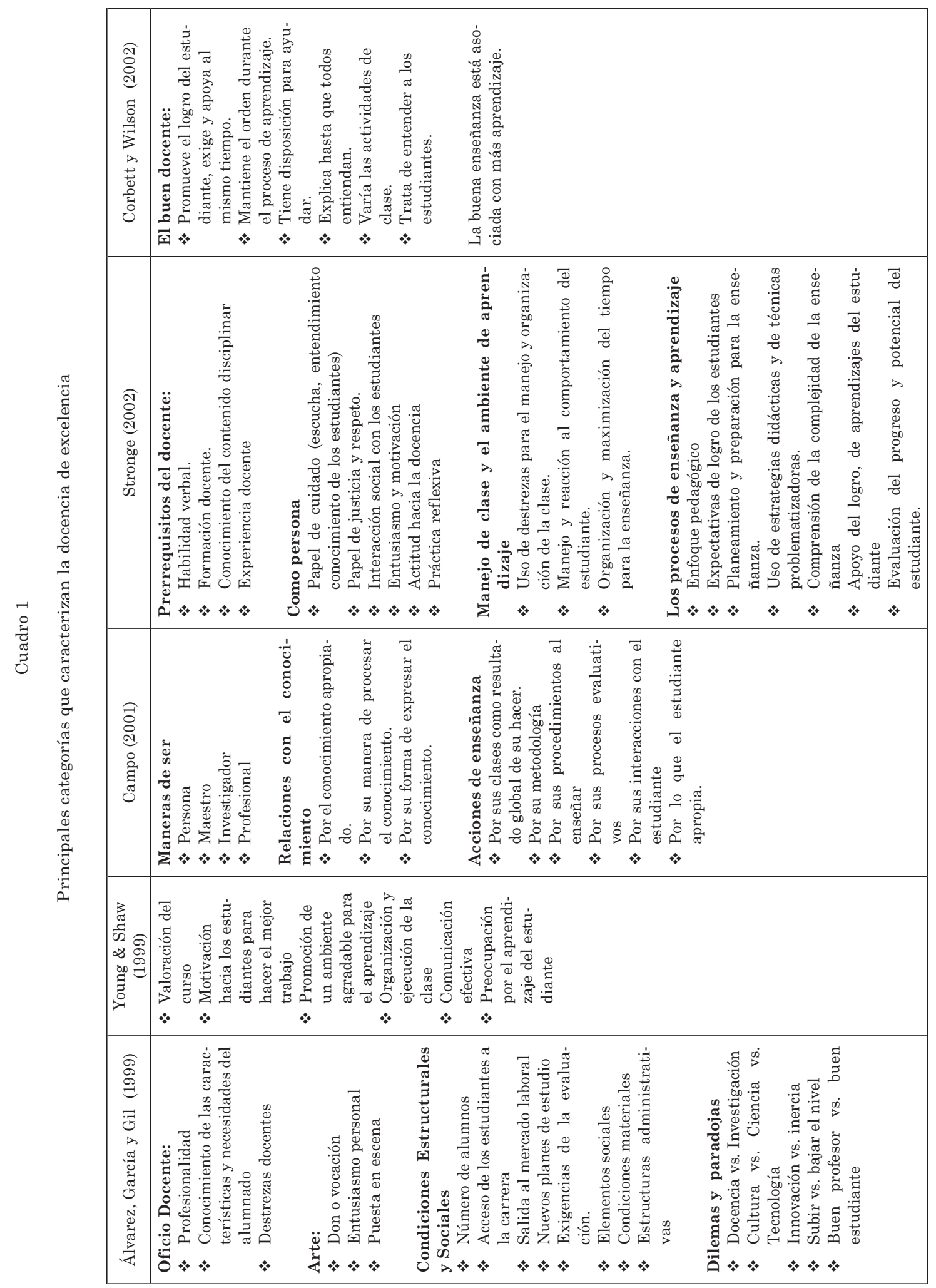


en dos vías en la que ambos, docente y estudiante, se identifican como actuantes en el proceso educativo. Constituye el reconocimiento de la existencia del "otro" con características particulares y requerimientos específicos.

La identificación y comprensión de los aportes, dudas y problemas del estudiante supone una habilidad de parte del docente para la comunicación que favorece la participación de los estudiantes: "La habilidad para la comunicación con los alumnos supone la capacidad para establecer cauces de comunicación, eliminar barreras y humanizar la relación personal entre el profesor y los alumnos". (Álvarez, García y Gil, 1999).

La comprensión de parte del docente lleva al entendimiento de las preocupaciones del estudiante en el espacio de aula y del contexto que incide en el proceso de aprendizaje. En el caso del estudiante universitario, los contextos pueden diferir sustancialmente: estudiantes madres, estudiantes que se trasladan a residencias estudiantiles y dejan su lugar de procedencia, niveles de formación, entre otros. Estas diferencias marcan la existencia de requerimientos de los estudiantes, también diversos (i.e. atención durante y fuera de la clase, individualmente o bien en pares, entre otros) que demandan la comprensión de las características y estilos de aprendizaje de estos. (Corbette, 2002).

Tales condiciones, en algunos casos, manifestadas como carencias formativas en los discentes, se deben identificar y comprender, ya que se expresan en términos diversos entre los estudiantes y entre otros, forman parte de los aspectos que inciden en la dinámica propuesta para la clase: “... (Los estudiantes)... aprecian especialmente aquellos docentes que hacen un esfuerzo por ver más allá del comportamiento de los estudiantes y comprenden quiénes son ellos realmente". (Ob. cit, 20).

Con el propósito de identificar aquellos aspectos que interfieran en los procesos pedagógicos, el docente requiere de mantener una apertura hacia la comunicación entre él o ella y los estudiantes, que a su vez, le permita acceder al conocimiento de estos últimos. La naturaleza de este conocimiento también se reconoce como diversa, puesto que no sólo incluye lo anteriormente descrito, sino que además supone considerar el contexto socio-histórico en el que se desarrolla el estudiante y su desarrollo como humano con capacidades para la construcción de saberes. Este conocimiento facilita la toma de decisiones en los procesos de aprendizaje, clima de clase, atención a procesos culturales fuera de la institución educativa, los límites de acción del docente y del estudiante y los problemas que la enseñanza universitaria les plantea a los docentes y estudiantes y las actitudes que han mostrado para enfrentarlos.

Las interacciones sociales con los estudiantes definen la relación del profesor universitario en los ámbitos formales académicos y en los ámbitos informales. Varias expresiones cotidianas de docentes universitarios recogen inquietudes al respecto, puesto que la naturaleza de estas interacciones interfieren en su percepción de autoridad del profesor ${ }^{10}$. El análisis de los reportes señala que los docentes "excelentes" se caracterizan por una actitud hacia el estudiante basada en un interés por el desarrollo personal en la esfera afectiva, un trato respetuoso y empático y, por último, la disponibilidad para el estudiante por su apertura y compromiso con la tarea de aprendizaje (Campo, 2001. Estas interacciones muestran una habilidad para relacionarse con los estudiantes y plantear conexiones afectivas positivas, lo cual supone acciones que van más allá de las paredes del aula y van a jugar un papel significativo en la promoción del logro de los estudiantes: "Los docentes que ofrecen apoyo, con frecuencia integran estudiantes que previamente han estado renuentes a participar en el trabajo del aula" (Corbette, 2002:20). 


\section{b. $\quad$ Valores asociados a la relación docente $y$ estudiante}

Más allá de las demostraciones de afecto, los docentes con alto grado de efectividad reflejan distintos grados de valores humanos ${ }^{11}$ en su relación con los estudiantes. Los valores de respeto y justicia son señalados con frecuencia por los estudiantes y se manifiestan en la escucha de opiniones, en la comprensión de lo expresado, la atención particular de la situación que se presente y en el trato equitativo en las condiciones de género, etnia, religión o condición sociocultural. (Stronge, 2002).

Las manifestaciones de respeto se conciben en un trato que asume al estudiante como persona con sentimientos, temores e incertidumbres cuando se proponen acciones de interacción en el aula. No obvia que existen situaciones particulares que median en el desarrollo del logro de las intenciones educativas en cada estudiante, por ejemplo la procedencia, las características de la formación previa a la universitaria, su condición de género, entre otras.

\section{c. Promoción del aprendizaje en los estudiantes}

Este rasgo se asocia con las formas utilizadas por el docente para generar entusiasmo y motivación por el logro de las tareas propuestas como intención educativa. Young \& Shaw (1999) incluyen la motivación como un predictor y rasgo fundamental en los perfiles del docente universitario efectivo obtenidos en su investigación. ${ }^{12}$ Cuando nos referimos al entusiasmo, se hace alusión al apasionamiento y emoción sentidos al enseñar y por el aprendizaje del contenido. ${ }^{13}$ Este entusiasmo es percibido como un importante factor de la motivación del estudiante, entendida como la disposición para atender, escuchar y mostrarse interesado por el aprendizaje de determinado contenido. (Campo, 2001; Stronge, 2002). Es necesario aclarar que la motivación es intrínseca al ser humano por lo que el docente no puede motivar a sus estudiantes sino generar ambientes propicios para que los estudiantes generen motivación hacia el objeto que estudia. No debe confundirse con ambientes de estímulo (como se conciben en el enfoque psicológico conductista) sino más bien con espacios retadores y problematizadores. El reconocimiento de la motivación no se queda en la generación de acciones por parte del docente solamente, sino que además se refiere a la implicación del estudiante en su proceso de construcción de conocimiento y a la búsqueda del equilibrio entre facilitar este aprendizaje frente a problematizarlo. (Alvarez, García y Gil, 1999).

\section{d. Disposición hacia la docencia}

La actitud mostrada por el profesor hacia el quehacer que desarrolla en el aula y como docente se orienta hacia dos intereses:

Por el aprendizaje de los estudiantes: El docente asume la responsabilidad en el logro de aprendizajes del estudiante que atiende. Así el éxito y el fracaso los vive como frutos de su actividad pedagógica. (Campo, 2001). Por su propio aprendizaje: Invertir en su proceso de mejoramiento profesional incluye la participación en actividades de actualización sobre su disciplina y acerca de lo pedagógico: "Ellos sirven como un poderoso ejemplo de aprendices de toda la vida que encuentran distintas vías para desarrollarse profesionalmente" (Stronge, 2002:20). Un interés como este evidencia una búsqueda constante y una apertura a la novedad, en un nivel crítico, pues todo contenido sufre un "proceso de aceptación". De acuerdo con Campo (2001) el profesor no enseña nada que no haya comprendido y aceptado, esto es, lo que enseñan es un contenido del cual se han apropiado: 
No se trata solamente de poseer información, ni de seguir un texto, ni de tener buena memoria, sino, justamente, del sentido que los maestros le dan al conocimiento porque lo han hecho parte de sí mismos. (Campo, 2001:118).

\section{La dimensión disciplinar}

La dimensión disciplinar se asocia con el profesor por su relación con el conocimiento de su disciplina. Empero, una evaluación del docente deberá considerar que para su valoración la opinión de los estudiantes aquí no es suficiente, pues los niveles de profundización y manejo disciplinar no les permiten la emisión de juicios de valor al respecto, lo cual sí podrían hacer docentes pares o colegas del profesor. Esto último debe ser de especial atención para los procesos de evaluación en los ámbitos institucionales en virtud de que requieren procesos sistemáticos y colaborativos, esto es, considerar diversas fuentes para la valoración de los docentes.

Existen, sin embargo, esferas de la dimensión disciplinar que atañen y son reconocidas por los estudiantes por su conexión con lo pedagógico, por ejemplo, el dominio del contenido disciplinar, o sea el manejo que refleja en la estructura conceptual y procedimental de lo que enseña está libre de improvisación y tiene pertinencia con el entorno cultural y específicamente de la profesión.

La investigación sobre esta dimensión incluye los factores asociados que plantean un dominio de la estructura conceptual de la disciplina. Young \& Shaw (1999) reportan que el conocimiento del contenido disciplinar obtuvo mayor media aritmética, 8,06, en una escala de 1 a 10 como predictor de la efectividad docente. Sin embargo, en los perfiles propuestos por los autores no aparece ninguna característica asociada a esta dimensión. Mientras que Marsh (1990) reporta que "la amplitud de conocimientos" muestra correlaciones entre 0,454 y 0,327 con el valor del aprendizaje, la claridad y organización de la clase, las interacciones grupales, con la interacción personal, amplitud de los conocimientos, las evaluaciones y calificación, las lecturas y trabajos asignados y sólo presenta una correlación baja de 0,106 con la carga de trabajo y su dificultad.

El conocimiento que el profesor tiene del objeto de estudio que enseña le permite reconocer los obstáculos epistemológicos que los estudiantes pueden presentar para aprehenderlo y así tomar decisiones pedagógicas según sea el caso; tiene mayor posibilidad de involucrar a los estudiantes en discusiones significativas, focalizar los puntos de mayor atención y las relaciones conceptuales que sostienen las categorías que explican profesionalmente el objeto de estudio. (Stronge; 2002, Álvarez, García y Gil; 1999).

Este dominio también evidencia una aproximación crítica hacia el conocimiento disciplinar. La capacidad crítica y analítica favorece el uso de analogías, de distinciones y de síntesis. (Campo, 2001) las cuales en el contexto sólo se identifican si existe una acción reflexiva sobre los conocimientos.

La relación de dominio del conocimiento a la cual se refieren los estudiantes incluye el bagaje y apropiación de los saberes disciplinares y además los "algoritmos" que el docente muestra en su relación con esos saberes. Por ello se presentan los procedimientos para clarificar los conocimientos y los valores asociados a este saber, aunado a esto último se debe agregar que también está implícita la jerarquización de conceptos (Vg. Cuando se habla de conceptos básicos) que según el imaginario colectivo de la disciplina se requiere para la formación profesional.

\section{La dimensión pedagógica}

La dimensión pedagógica viene a constituir aquellas visiones y acciones que permiten el logro de calidades y valores comportamentales que son objeto de 
determinada formación profesional, entendida como la visión que se concretará en la acción educativa. Esta sección se propone destacar aquellos rasgos que se incluyen en la tradición evaluativa del desempeño del docente universitario.

Marsh (1990) identifica nueve factores que caracterizan la efectividad docente, al menos siete se relacionan con la dimensión pedagógica, entre éstos, "la organización y claridad en la clase" presenta correlaciones entre 0,411 y 0,210 con el valor del aprendizaje; las interacciones grupales; con la interacción personal; amplitud de los conocimientos; las evaluaciones y calificación; las lecturas y trabajos asignados; sólo presenta una correlación baja de 0,04 con la carga de trabajo y su dificultad. Por su parte Cashin (1988) reportó que las correlaciones promedio con factores asociados a la categoría pedagógica son superiores a 0,44, mientras que las habilidades docentes alcanzan la correlación promedio más alta con 0,50 .

El conocimiento disciplinar en sí mismo es necesario, pero no suficiente para poder enseñarlo. Se requiere comprender la forma en que los estudiantes van a acceder y aprehender este conocimiento. Esto implica la identificación de las acciones que facilitan y pueden obstaculizar este proceso. (Stronge, 2002; Ferguson \& Womack, 1993; Collins, 2001). Esta comprensión se puede enfocar de distintas maneras. La primera de ellas es por su relación con los sujetos que participan en el acto educativo y la segunda por el papel que cumplen los elementos curriculares como los objetivos (intenciones educativas), el contenido, el método y la evaluación.

Los distintos abordajes evaluativos consideran variedad de denominaciones para caracterizar la acción educativa (aquí asumimos que lo educativo es consustancial a lo pedagógico, en tanto lo educativo es objeto de estudio de la pedagogía. En el cuadro 2 se pueden apreciar los factores identificados que estadísticamente explican la efectividad docente, en ellos se aprecian elementos de corte didáctico como las estrategias de enseñanza y elementos curriculares como la organización y planificación.

Distinguir las fronteras de lo pedagógico es nuevamente complejo para este caso, por lo que resulta indispensable aclarar que es necesario superar la denotación didáctica y reconocer la sustancia de lo pedagógico como lo educativo. Lo pedagógico se convierte en una gran sombrilla que conjuga los aportes de distintas disciplinas científicas para explicar el acto educativo, por ejemplo, la Didáctica estudia la enseñanza, el Currículo la selección, organización y planificación de la cultura hecho educativo y la Psicología, las teorías del aprendizaje y la motivación para este según la etapa de desarrollo humano, entre otras.

Algunas evaluaciones sobre el desempeño del docente universitario evidencian esta síntesis, empero, generalmente los rasgos relacionados con lo pedagógico son de naturaleza didáctica y curricular, como se puede apreciar en las siguientes categorías.

\section{a. El enfoque pedagógico}

Las acciones docentes están mediadas por una forma de comprender el acto educativo y los elementos que se conjugan en él, estas asumen una dimensión psicológica de los sujetos, la dimensión lógica que configura el camino para lograr las intenciones y la dimensión ontológica que permite el reconocimiento de la naturaleza del objeto que espera conocer los sujetos.

Estas comprensiones determinan las expectativas comportamentales que se ensayarán en el aula. Por esto constituyen entramados de ideas o presupuestos filosóficos, ideológicos, sociales y políticos que soportan y justifican la toma de decisiones en la actividad docente. Tres cuestiones básicas se responden, la primera tiene que ver con el papel del docente, en el que se reconocen distintas formas de expresión como "el transmisor de conocimientos; el 
Cuadro 2

Instrumentos y factores asociados con la efectividad en la docencia

Instrumento

Factores que miden la efectividad en la Docencia Universitaria

1. Instrumento de Esfuerzo de Frey (Frey, Leonard \& Beatty, 1975, Marsh, 1981, 1986)

2. Descripción del Estudiante sobre la enseñanza (The Student Description of Teaching: SDT) desarrollado por Hildebrand, Wilson y Dienst (1971)

3. Evaluaciones del Estudiante sobre la Calidad Educativa de Marsh (Student Evaluations of Educational Quality: SEEQ) (Marsh, 1982b, 1983, 1984, 1987)

4. El Instrumento SIRS del Estado de Michigan (Warrington, 1973)
Claridad de la presentación,

Carga de trabajo

Atención personal,

Discusión en la clase,

Organización y planificación

Evaluación , y

logros del estudiante

Aproximación analítica/sintética

Organización/claridad

Interacción del docente en el nivel grupal.

Interacción del docente en el nivel individual.

Entusiasmo/dinamismo

Aprendizaje/valor

Entusiasmo docente

Organización/claridad

Relación individual

Interacción grupal

Amplitud del conocimientos

Evaluaciones/calificación

Asignaciones/lecturas

Carga de trabajo/dificultad

Complejidad de la instrucción

Interés y actuación del estudiante

Interacción estudiante-docente

Exigencia del curso

Organización del curso

Fuente: Marsh, H.W, 1990.

de iniciador/motivador para la adquisición autónoma de conocimientos por parte del alumno; el de tutor del proceso de adquisición; el de innovador y cuestionador del conocimiento aceptado". (Álvarez, García y Gil, 1999:282).

El espacio universitario también se propone como un nicho para el tránsito de conocimientos entre docentes, estudiantes y administrativos. El docente universitario se ve obligado también a ampliarlo; innovar; cuestionar; incorporar contenido, preocupaciones, sensibilidades de distintos ámbitos; como se percibe en los binomios docencia- investigación y docencia-extensión social, entre otras exigencias.

La segunda cuestión tiene relación con el contexto. Ninguna consideración con respecto al docente universitario se resuelve sin considerar el contexto en el cual se desarrolla. Concebido como el ámbito cultural que incorpora el conjunto de influencias configurativas del actuar, el contexto define la necesidad de correlacionar la teoría y la práctica, por ejemplo, esta asociación se concreta en la búsqueda de la aplicabilidad crítica del contenido curricular en el "mundo externo" de situaciones culturales, 
profesionales o cotidianas (ob. cit). El contexto surge como cuestión según el enfoque pedagógico que asume el profesor. Así los enfoques pedagógicos que lo consideran superan la posición del profesor como transmisor de conocimientos, como vaciador de información. ${ }^{14}$

La última cuestión se refiere al aprendizaje del estudiante, lo que se apropia en el proceso de formación profesional. La expectativa del docente hacia el logro de aprendizajes se relaciona con la comprensión y la aplicación creativa más que con la repetición mecánica (Campo, 2001) en un camino de ascenso hacia la autonomía y valoración del sentido de los logros educacionales y profesionales que puede alcanzar el curso que desarrolla. La apropiación que hace el estudiante no es inerte, más bien lo orienta hacia el desarrollo del sentido crítico frente a su profesión y su implicación en la dinámica social.

\section{b. El enfoque curricular}

Es un rasgo distintivo del docente la previsión de elementos, actividades y momentos próximos de la intervención pedagógica. Esta previsión otorga el carácter intencional y efectivo de la tarea docente y le aseguran la consecución de su quehacer. Existe un nivel de concreción preescrito que se incorpora en la planificación, organización del programa, selección de contenidos, determinación de las estrategias didácticas y de los apoyos técnicos y materiales, así como de la identificación de la estrategia evaluativa.

El enfoque curricular asumido institucionalmente y por el docente, permite distinguir las pautas y obstáculos para el desarrollo de los planes de formación profesional. Se asume, por tanto, que existe una conciencia de lo que puede llegar a ser el quehacer en el aula, desde la vinculación y síntesis de los elementos curriculares (docentes, estudiantes, contexto, método, objetivos, entre otros) para el logro de las intenciones educacionales. La selección y análisis del material didáctico, pasando por la determinación de estrategias para la enseñanza hasta los procesos evaluativos como camino para la autorregulación docente, son considerados significativos por su carácter profesional y pragmático.

\section{c. Elementos de naturaleza didáctica}

La enseñanza como acción se distingue por las formas de actuación del docente, las expectativas de logro de aprendizajes por parte de sus estudiantes, el desarrollo de contenidos, la estrategia didáctica como tal y la claridad expositiva del docente.

La didáctica de los docentes se reconoce en su "puesta en escena" (Campo, 2001). Las características didácticas de un docente excelente lo identifican con diversas prácticas, la primera de ella es la búsqueda de lo innovador. Lo variado es un adjetivo significativo que explica la presencia de novedades en las estrategias didácticas, estas últimas sugieren nuevos retos y altos niveles de participación en el quehacer global del espacio de aula como en el uso de nuevos recursos y materiales de orden didáctico.

Existe una distinción importante al ambiente generado por el docente para el logro de aprendizajes, el cual está íntimamente ligado con el carácter de las estrategias didácticas utilizadas:

Las clases de los mejores profesores son amenas, participativas, organizadas, novedosas, divertidas a las que "da gusto ir". Ellos mantienen el control de la clase, son rigurosos, imprimen entusiasmo y generan interés y agrado en sus alumnos. (Ob. cit: 120).

El uso de estrategias didácticas que se proponen problematizar y el desarrollo de pensamiento crítico se conciben como formas detonadoras de participación, pero a la vez de niveles superiores de desempeño que buscan trascender el dato en sí mismo. 
Por ello un ambiente coercitivo no decanta en niveles de participación y de reconocimiento del logro de mejores expresiones acerca del contenido profesional.

El uso de estrategias didácticas de esta naturaleza le permite al docente desarrollar la capacidad de determinar las carencias formativas y conceptuales de los estudiantes y luego, traducir las complejidades del objeto disciplinar para hacerlo enseñable y lograr su aprehensión por parte del estudiante. En este proceso la capacidad comunicativa es fundamental como parte del acto pedagógico.

La dinámica que envuelve el acto educativo está sustentada por interacciones frecuentes en las cuales todo hacer y no-hacer tienen una denotación y connotación mediada por la cultura y ofrece distintos mensajes. Sin embargo, el contenido disciplinar ofrece dificultades y una complejidad propias del conocimiento formal que requiere un tratamiento para su comunicación. La habilidad verbal para Stronge (2002), claridad expositiva para Campo (2001) y Álvarez, García y Gil (1999) se define como una capacidad para la comunicación efectiva que permite explicaciones con sentido, hasta que todos comprendan. (Corbette, 2002, Campo, 2001).

La incorporación de distintas expresiones acerca de los núcleos temáticos con un uso de discursos inteligibles permite la transferencia de ideas a otros planos y, por tanto, la comprensión de las redes conceptuales del contenido disciplinar. El proceso de hacer enseñable un contenido del objeto de estudio de la disciplina, exige una comprensión epistemológica, heurística, ontológica y axiológica de éste, por ello no puede omitirse la relación de la comunicación efectiva con la dimensión disciplinar. Así también, las posibilidades de comunicación se dan en espacio de respeto y atención al otro, por lo que no se puede tampoco negar la relación con la dimensión personal. Es necesario comprender la comunicación como un núcleo que articula los tres planos dimensionales para favorecer los procesos educativos que se buscan.
La capacidad comunicativa permite la aproximación pedagógica del contenido disciplinar con el estudiante, traducir su complejidad en espacios de acceso flexible para él y la posibilidad de constituirse en sujetos que interactúan en ámbitos emocionalmente equilibrados para la construcción de la estructura conceptual disciplinar.

\section{Consideraciones finales}

Cuando se reconoce la naturaleza educativa del quehacer del docente universitario es cuando la comprensión de su praxis se complejiza. La mayoría de los retos que se demandan al profesor o profesora universitaria, sitúan esta comprensión en el análisis de la multidimensionalidad que es evidente a partir del estudio de las realidades. De este carácter multidimensional emerge como puntos centrales el reconocimiento de lo personal, lo disciplinar y lo pedagógico y por tanto, la necesaria atención que debe prestarse a sus interrelaciones. La distinción surge como necesidad para poder aproximarse sistemáticamente al estudio del docente universitario y poder así proponer bases para apoyar su desarrollo académico. No obstante se deja claro que la selección de los docentes universitarios y su proceso de desarrollo académico no puede limitarse a lo disciplinar, deberá asumir lo personal y lo pedagógico, de hecho lo pedagógico no existe si no es al interior de una disciplina y lo pedagógico propone formas de interacción personal: "La relación del contenido con la pedagogía no es paralela, es intrínseca, inclusiva e inseparable y propone vías de interacción interpersonal, entre los distintos artificios disciplinarios que se crean para su enseñabilidad". (Francis, 2005:14).

La profundización en el análisis de estas dimensiones repercute también en las formas simplistas en que algunas de estas son expresadas a la hora de elaborar perfiles. Los "listados de características" 
no dejan entrever las relaciones intrínsecas que fluyen entre unas y otras. Las propuestas de desarrollo académico requieren analizar críticamente estos formatos para no convertirlos en simples "check list" para dar orientación a los procesos formativos y evaluativos. La complejidad del acto docente se ve demostrada en las interrelaciones de dichas dimensiones, y por ello, es necesario proponer metodologías que permitan evidenciar y reconstruir desde la dialéctica que se muestra entre las dimensiones del docente y su vínculo con las comunidades académicas.

Cualquier proyecto que busque apoyar la formación académica del docente universitario enfrentará como tarea la distinción e interrelación de tales dimensiones. En aras de buscar la superación de enfoques tradicionales en el aula hacia enfoques formativos de mayor pertinencia, según la lectura de las actuales demandas sociales, es urgente promover espacios de reflexión-acción que develen estas relaciones a partir de las sistematizaciones de investigaciones como las consideradas en este artículo.

Finalmente, es necesario constatar si el encaje de los sistemas de expectativas e intenciones del docente con los de los estudiantes como forma de conceptuar la excelencia de los docentes también se ve reflejado en los logros esperados, o sea, los conocimientos construidos. Queda como camino abierto indagar y analizar la asociación entre estas dimensiones y el rendimiento académico de los estudiantes, este último visto como el empleo de estrategias cognitivas y procesos de pensamiento para la transformación de la conciencia intelectiva.

\section{Notas}

1. Este artículo forma parte de la depuración teórica-conceptual del proyecto de investigación $\mathrm{N}^{\circ}$ 724-A5-555 Los saberes pedagógicos del docente universitario: una mirada a los profesores valorados excelentes por sus estudiantes del cual la autora es la investigadora principal.
2. Ver los reportes de González, Ferres y Gutiérrez (2001), Contreras (1995), Hawe (2002) y Sarama (2002) Castle (2001), Goddard (2001), Mayor (1996), Varela, Petra, González y Ponce de León $(1998,2000)$.

3. Para efectos de clasificar los estudios de investigación, se consideró los supuestos planteados por Habermas (1990).

4. Ídem anterior.

5. Vease los estudios de Danielson \& MGreal 2000; Marzano, Pickering \& Pollock 2001; Tell, 1999; Rowley 1999; Scherer 2001; Gil 2000; Morán 2003; Díaz e Inclán 2001.

6. Ver al respecto los estudios de la relación de la docencia efectiva con la investigación de Marsh \& Hattie (2002), los reportes señalan las variables que se correlacionan con las calificaciones de los docentes universitarios y los factores que la explican en la dimensión tecnológico-instrumental, el análisis de sesgo de Cashin (1988), Marsh (1987, 1990, 1997), y los análisis de evaluaciones desarrolladas por Rice (1998), Hujber (1998), Ledic, Rafajac y Kovac (1999), Young \& Shaw (1999), los estudios sobre la efectividad en los procesos de formación docente universitaria, sobre todo, el papel de lo pedagógico de Garet, Porter, Desimone, Birman \& Suk Yoon (2001), y el estudio sobre estrategias para una pedagogía efectiva de Tsui (2002).

7. Esto se refleja en las investigaciones de Campo (2001), Álvarez, García y Gil (1999) Cotton (2000), Darling-Hammond \& Thoreson (2001), Emmer, Evertson, y Anderson (1980) Jonson (1977), Millar, McKenna y McKenna (1998), Peart y Campbell (1999), Wang, Haertel y Walberg (1994) Tsui (2002).

8. Young \& Shaw (1999) aplicaron la función de análisis discriminante para determinar en el análisis de los resultados un subconjunto de ítemes predictores que diferenciara los docentes efectivos de los inefectivos.

9. Se refiere a frases escuchadas en las sesiones de reflexión que se desarrollan dentro de los cursos de Didáctica Universitaria, del Departamento de Docencia Universitaria de la Facultad de Educación, UCR.

10. Según Schwartz (2003:54) existe un gran acuerdo en la bibliografía en distinguir cinco rasgos que definen un valor. "Un valor es 1) una creencia; 2) que pertenece a fines 
deseables o a formas de comportamiento; 3) que trasciende las situaciones específicas; 4) que guían la selección o evaluación de comportamientos, personas y sucesos; y 5) que se ordena por su importancia relativa a otros valores para formar un sistema de prioridades de valores".

11. En la investigación realizada por Young \& Shaw se señala que la motivación obtuvo un coeficiente de determinación de un $\mathrm{r}^{2}=0,796$.

12. Nos referimos al contenido desde la didáctica o sea como el entramado de valores, habilidades, destrezas y componentes teóricos, epistemológicos, heurísticos y axiológicos que definen un objeto de estudio.

13. No es bueno aquí confundir estrategia didáctica con el enfoque del papel del docente. Ser magistral, por ejemplo, permite la problematización tanto del texto oral como del escrito, gráfico y hasta del texto ausente, y no sugiere necesariamente un vaciado de información.

\section{Referencias bibliográficas}

Álvarez Rojo, Víctor; García Jiménez, Eduardo y Gil Flores, Javier. (1999). "La calidad de la enseñanza universitaria desde la perspectiva de los profesores mejor valorados por los alumnos" Revista de Educación 319 España.

Berger, Peter \& Luckmann, Thomas. (1984). La construcción social de la realidad Argentina: Amorrortu Editores.

Campo Vázquez, Rafael. (2001). Caracterización de una excelente práctica docente universitaria para estudio de caso en la Pontificia Universidad Javeriana, Bogotá Colombia. Tesis Doctoral Universidad de Costa Rica.

Cashin, William. (1988). "Student Ratings of Teaching: A Summary of Research" IDEA Paper $N^{\circ} 20$ Center for Faculty Evaluation \& Development Division of Continuing Education: Kansas State University.
Castle, Joyce B. (2001). "Teacher Education and Leadership for Change: Exploring Faculty Perspectives". Alberta Journal Educational Research 47 [En red] Disponible en dirección electrónica:

http://www.education.ualberta.ca/ educ/journals/ajer/4 7 files/ 47(2)files/47(2).html

Collins, Robyn. (2001). "Best Practices in Teaching and Learning: What Does the Research Say?" [En red] Connections (AISQ), Queensland. Disponible en http://www.curriculum.edu.au/ scis/connect/cnetw02/cnet41in.htm

Contreras, Montes de Oca, Ileana. (1995). "El quehacer diario del profesor de matemática en la educación secundaria: Algunos resultados de su estudio" Revista Educación 19(1).

Corbett, Dick \& Wilson, Bruce. (2002). "What urban student say about good teaching" Educational Leadership 60 (1).

Danielson, Charlotte \& McGreal, Thomas L. (2000). Teacher Evaluation:Toenhance profesional practice Alexandría, Va. EE.UU. Association for Supervision and Curriculm Development.

Darling-Hammond, Linda; Berry, Barnet, \& Thoreson, Amy. (2001). "Does teacher certification matter? Evaluating the evidence" Educational Evaluation and Policy Analysis 23 (1).

Díaz Barriga, Ángel e Inclán, E. Catalina. (2001). "El docente en las reformas educativas: sujeto o ejecutor de proyectos ajenos". Revista Iberoamericana de Educación 25.

Emmer, Edmund; Evertson, Carolyn \& Anderson, Linda. (1980). "Effective Classroom Management at the 
Beginning of the School Year". The Elementary School Journal 80 (5).

Ferguson, Patrick \& Womack, Sid. (1993). "The impact of Subject Matter and Education Coursework on Teaching Performance" Journal of Teacher Education 44 (1).

Francis Salazar, Susan. (2005). "El conocimiento pedagógico del contenido como caegoría de estudio de la formación docente" [En red] Actualidades Investigativas en Educación 5 (2) Disponible en dirección electrónica http://revista.inie.ucr.ac.cr/ articulos/2-2005/archivos/ conocimiento.pdf

García-Valcárcel Muñoz, Ana. (2001). Didáctica Universitaria Madrid, España: Editoral Muralla.

Garet Michel, Porter; Andrew Desimone, Laura; Birman, Beatrice \& Yoon, Kwang Suk. (2001). "What makes professional development effective? Results from a national sample of teachers". American Educational Research Journal 38 (4).

Gil, Manuel. (2000). "Los académicos en los noventa: ¿actores, sujetos, espectadores o rehenes?" Revista Electrónica de Investigación Educativa 2 (1) [En red] Disponible en dirección electrónica http://redie.ens.uabc.mx/vol2no1/ contenido-gil.html.

Goddard, Tim. (2000). "Teaching in Turbulent Times: Teachers' Perceptions of the Effects of External Factors on Their Professional Lives" Alberta Journal Educational Research 46 [En red] Disponible en dirección electrónica:

http://www.education.ualberta.ca/ educ/journals/ajer/46files/ 47(2)files/46(2).html
González Carbajal, Eleuterio; Petra Micu, Ileana; Varela, Margarita y Ponce de León, María Eugenia. 1998 "La Construcción semántica del concepto de profesor en docentes de medicina" Revista de la Educación Superior 107 ANUIES Julio-Agosto. [En red] Disponible en página electrónica http://web.anuies.mx/anuies/revsup/ res107/text1.html\#i

González Carbajal, Eleuterio; Petra Micu, Ileana; Varela, Margarita y Ponce de León, María Eugenia. (2000). “Análisis semántico del concepto de enseñanza de profesores de medicina". Revista de la Educación Superior 116 ANUIES Julio-Agosto [En red] Disponible en página electrónica http://web.anuies.mx/anuies/revsup/ res116/text1.html\#i

Habermas, Jürgen. (1995). Conocimiento e interés Educacio. Materials de Filosofia. Universitat de València.

Hawe, Eleaonor. (2000). "Assessment in a pre-service teacher education program: the rhetoric and the practice of standards-based assessment. AsiaPacific Journal of Teacher Education 30 93-106 [En red] Disponible en https://www.acer.edu.au/scripts/ library/request/docreq0 1. php?document_id=115729

Jonson, Bob. (1977). "An organizational Analysis of Multiple Perspectives of Effective Teaching: Implications for Teacher Evaluation". Journal of Personnel Evaluation in Education 11.

Ledic, Jasminka; Rafajac, Branko \& Kovac, Vesna. (1999). "Assesing the quality of university teaching in Croatia". Teaching in Higher Education 4 (2) Texto Completo Proquest Company. 
Marsh, Herbert. W. \& Hocevar, Dennis. (1990). The Multidimensionality of Students' Evaluations of Teaching Effectiveness: The Generality of Factor Structures Across Academic Discipline, Instructor Level, and Course Level [En Red] Disponible en dirección electrónica

http://www.psychologie.un-bonn.de/ online-documents/lit-oth.htm

Marsh, Herbet. W. \& Roche, Lawrence. A. (1997). "Making Students' evaluations of teaching effectiveness effective: The critical issues of validity, bias and utility" American Psychologist November. [En Red]

http://www.dartmouth.edu chance/ course/Syllabi/97Dartmouth/day-14/ eval-2.pdf

Marsh, Herbert. W. \& Hattie, John. (2002). "The Relation between research productivity and teaching effectiveness" The Journal of Higher Education 73 (5).

Marzano, Robert; Pickering, Debra, \& Pollock, Jane. (2001). Classroom Instruction that works Researchbased Strategies for Increasing Student Achievement ASCD Virginia USA.

Mayor Ruiz, Cristina. (1996). "Las funciones del profesor universitario analizadas por sus protagonistas. Un estudio atendiendo al grupo de titulación y los años de experiencia, en la Universidad de Sevilla." Revista Electrónica de Investigación y Evaluación Educativa 2. [En red] Disponible en http://www.uv.es/RELIEVE/ art_pub1.htm

Millar, John; McKenna; Micheal \& McKenna, Beverly. A. (1998). "A Comparison of Alternatively and
Traditionally Prepared Teachers" Journal of Teacher Education 49(3).

Morán Oviedo, Porfirio. (2003). "La relación pedagógica, eje para transformar la docencia”. Revista Electrónica de Investigación Educativa 5 (1) [En red] Disponible en dirección electrónica http://redie.ens.uabc.mx/vol5no1/ contenido-moran.html

Peart, Norman A. \& Campbell, Frances. A. (1999). "At - Risk Students' Perceptions of Teacher Effectiveness". Journal for a just and Caring Education 5 (3), 269-284.

Quirós Rodríguez, Tito y Jiménez Fallas, Kemly. (1991). El Perfil del docente para la Universidad de Costa Rica. Proyecto 724-89-021 IIMEC. Universidad de Costa Rica Manuscrito no Publicado. Universidad de Costa Rica.

Rice, Ronald; Stewart, Lea \& Hujier, Michel. (1998). "Extending the domain of Teaching effectiveness assessment" . [En Red] Disponible en http://www.scil.rutgers.edu/ rrice/ teachcon.htm

Robbins, Stephen. (1994). Comportamiento organizacional: conceptos, controversias y aplicaciones. 2ed. Prentice Hall Hispanoamericana México.

Rowley, James. (1999). "The Good Mentor" Educational Leadership 56 (8).

Sarana, Julie. (2002). "Listening to teachers: Planning for profesional development". Teaching Children Mathematics 9. Texto Completo: Proquest Company.

Scherer, Marge. (2001). "Improving the quality of the teaching force". Educational Leadership 58 (8). 
Schwarts Shalom. (2001). Psicología de los valores humanos. Desarrollos teóricos, metodológicos y aplicados. Ros, María y Gouveia, Valdeney. (Ed) Biblioteca Nueva. Madrid.

Stronge, James. (2002). Qualities of Effective Teachers. Alexandría, Va. EE.UU. Association for Supervision and Curriculm Development.

Tell, Caroll. (1999). "Renewing the profession of teaching: A conversation with John Goodlad" Educational Leadership 56 (8).

Torres, Rosa. (1998). Nuevo Rol del Docente: ¿Qué modelo de formación, para qué modelo educativo? [CD- ROM]. Curso Doctorado Educación: Educación Desarrollo de la Educación y la investigación en el contexto latinoamericano. (edal 136).

Tsui, Linda. (2002). "Fostering Critical Thinking through Effective
Pedagogy" The Journal of Higher Education 73 (6).

UNESCO. (1998). Debate temático La formación del personal de la Educación Superior: Una misión permanente Conferencia Mundial sobre Educación Superior: La Educación Superior en el siglo XXI Visión y Acción, UNESCO.

Venegas Renauld, María Eugenia. (1995). La función docente en la Universidad de Costa Rica. El caso de las y los docentes de las áreas de Ciencias Básicas y Ciencias Sociales. Tesis de maestría. Universidad de Costa Rica, Costa Rica.

Wang, Margaret; Haerte, Geneva \& Walberg, Herbert. (1994). "What helps students learn?" Educational Leadership 51 (4).

Young, Suzanne \& Shaw, Dale. (1999). "Profiles of Effective College and University Teachers" Journal of Higher Education 70 (6). 
